\title{
Lymph node enlargement after a single massive infusion of iron dextran
}

\author{
G. THEODOROPOULOS, A. MAKKOUS, AND M. CONSTANTOULAKIS1 \\ From the First University Clinic of Internal Medicine, \\ King Paul's Hospital, Athens
}

SYNOPSIS Painful lymphadenopathy developed in eight female patients after massive infusions of iron dextran for sideropaenic anaemia. Fever, arthralgias, and malaise were also features of the clinical picture. The histology of one lymph node is described and changes in it are discussed in relation to the pathogenesis of the syndrome reported.

During the past four years an iron-dextran ${ }^{2}$ complex has been used fairly extensively as a single massive infusion in the treatment of certain sideropaenic anaemias (Basu, 1963; Marchasin and Wallerstein, 1964; Bonnar, 1966; Maschas, Constantoulakis, Floras, and Makkous, 1966; Theodoropoulos, Constantoulakis, Zevgolatis, and Phocas, 1966; Symposium, 1967). This type of treatment has also been used by us in 250 patients who were suffering from sideropaenic anaemia of variable aetiology but mainly resulting from disorders of the gastrointestinal tract. The results of the treatment were very satisfactory and in all patients, except those who continued to bleed the anaemia was corrected within 30 days. Side effects with this method of iron infusion are not rare but, with a few exceptions, they are mild and transient.

The occurrence of lymph node enlargement in females is described as an unexpected side effect that has not been previously reported, although an iron-dextran complex has been infused in massive doses in several thousand patients.

\section{CASE HISTORIES}

CASE 1 D.K., aged 49 years, had anaemia from bleeding haemorrhoids. She was given a single infusion of $1,600 \mathrm{mg}$ iron-dextran complex diluted in $1,000 \mathrm{ml} 5 \%$ glucose. Twenty hours later the patient complained of arthralgias, headache, general malaise, became sick, and had a high temperature. The next day there was generalized, painful enlargement of lymph nodes that subsided four days later. The other symptoms also disappeared within four days. The size of the nodes was variable but the ${ }^{1}$ Address requests for reprints to Dr M. Constantoulakis, 4 Alkiviadou street, Athens (109), Greece.

${ }^{2}$ Imferon.

Received for publication 11 January 1968. largest one was $2 \mathrm{~cm}$. Forty-eight hours after the appear- $\bar{C}$ ance of the lymph nodes, one of them was removed for histology. The response to the anaemia was remarkably good.

CASE 2 A.M., aged 36 years, had post-gastrectomy sideropaenic anaemia. The patient received $1,500 \mathrm{mg}$ iron-dextran in $1,000 \mathrm{ml} 0.9 \% \mathrm{NaCl}$ as a single infusion. Three hours after completion of the infusion the patient complained of generalized arthralgias, $\frac{\circ}{\varnothing}$ headache, rigor, and a high temperature. All symptoms $\mathscr{\mathcal { Q }}$ subsided within 24 hours. Three days after the infusion $\overrightarrow{\vec{O}}$ of iron, generalized, painful lymph node enlargement appeared which lasted approximately 20 days. The size of the nodes was variable, the largest being $3 \mathrm{~cm}$. The response to the anaemia was satisfactory.

CASE 3 A.T., aged 32 years, had post-haemorrhagic sideropaenic anaemia from a bleeding duodenal ulcer. 3 She received $1,500 \mathrm{mg}$ iron-dextran in $1,000 \mathrm{ml} 5 \%$ glucose. On the third post-infusion day, the patient complained of severe arthralgias, muscle pains, general malaise, and had a high temperature. One day later, generalized, painful enlargement of the lymph nodes $\square$ was noticed, which subsided within five days togethero with the other symptoms. The response to the anaemia
was rapid and complete.

CASE 4 D.C., aged 28 years, had a possibly idiopathic N sideropaenic anaemia. The patient received $2,000 \mathrm{mg}$ iron-dextran in $1,000 \mathrm{ml} 0.9 \% \mathrm{NaCl}$. On the third post infusion day arthralgias, backache, malaise, and aco moderate increase of temperature appeared. At the same $\Phi$ time, painful enlargement of the cervical and inguinal $\stackrel{\oplus}{+}$ lymph nodes was noticed. The patient's symptoms 0 subsided within two days but the lymph nodes remained 0 palpable for eight days. The anaemia was completely corrected within 25 days.

CASE 5 P.L., aged 45 years, had post-haemorrhagic 믐 sideropaenic anaemia from bleeding duodenal ulcer. 
Iron-dextran, $2,000 \mathrm{mg}$ in $0.9 \% \mathrm{NaCl}$, was given. On the third post-infusion day the patient complained of diffuse arthralgias and two days later slight, painful, generalized enlargement of the lymph nodes was noticed that subsided in four days. Correction of the anaemia was rapid and complete.

CASE 6 G.K., aged 34 years, had a possibly idiopathic sideropaenic anaemia, treated with $1,250 \mathrm{mg}$ of irondextran. Two days later the patient complained of arthralgias and had a moderate increase in temperature. On the eighth post-infusion day two nodes were noticed on the right lateral cervical area. They were $2 \mathrm{~cm}$ in size and painful. The nodes disappeared in four days and the response to the treatment was satisfactory.

CASE 7 I.P., aged 35 years, had post-haemorrhagic sideropaenic anaemia from metrorrhagias. The patient received $2,000 \mathrm{mg}$ iron-dextran as a single infusion and next morning she complained of arthralgias, headache, and general malaise, but the temperature remained normal. On the third day one small painful node was palpated in the right lateral cervical chain that disappeared three days later.

CASE 8 G.L., aged 19 years, had post-haemorrhagic anaemia from bleeding duodenal ulcer. She received $1,500 \mathrm{mg}$ iron-dextran without any side effect. On the eighth post-infusion day one large $(4 \mathrm{~cm})$ painful lymph node was palpated in the right inguinal area. The node regressed in three days.

\section{HISTOLOGY}

The lymph node removed was $2 \mathrm{~cm}$ in diameter. Its consistency was elastic and its colour whitish with some small yellowish or brown areas.

On sections stained with haematoxylin and eosin it was found that the normal architecture was maintained, there was no infiltration of the node capsule, and the lymph follicles were not hyperplastic. No inflammatory infiltration was seen. The sinuses were dilated and filled with reticuloendothelial cells which showed a significant hyperplasia. Some of the reticuloendothelial cells contained yellowish-brown, refractile granules which were thought to differ from the reddish brown colour of the haemosiderin deposits. Sections, stained with the Perls reaction for iron, showed that these granules had iron (Figs. 1 and 2).

Reticuloendothelial cells containing the above described yellowish-brown iron granules were also seen diffusily in the lymphoid parenchyma of the node either isolated or in clusters. Plasma cells were also significantly increased in the medulla. No germinal centres were seen, and the cortex did not show significant changes.

In conclusion, an abundant, iron-containing substance was found in the hyperplastic reticuloendo-

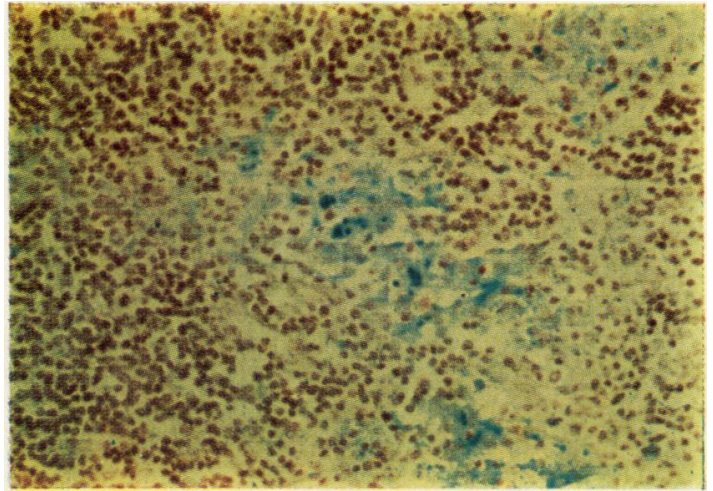

FIG 1. Section of the lymph node showing marked hyperplasia of reticuloendothelial cells and increase of plasma cells. Haematoxylin and eosin.

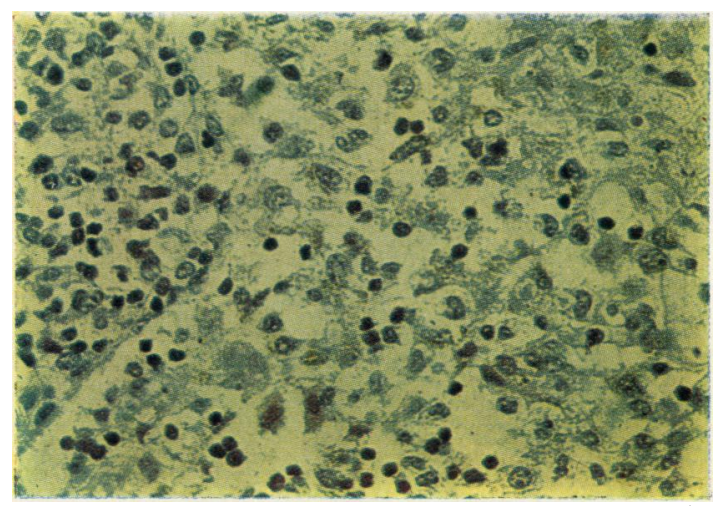

FIG 2. Section of the lymph node stained with the Perls reaction showing significant amounts of iron in the reticuloendothelial cells, especially in the sinus.

thelial cells, especially in the sinuses. Increased numbers of plasma cells were also seen in the medulla of the node.

\section{DISCUSSION}

All the above described cases of localized or generalized painful lymph node enlargement occurred in sideropaenic females treated with a single massive intravenous infusion of iron-dextran complex. In almost all cases the lymph node enlargement occurred two to five days after the iron administration, it was of short duration, with one exception, and general symptoms such as fever, arthralgias, and malaise preceded or appeared simultaneously with it. Both symptoms and lymph node enlargement subsided without specific treatment and did not interfere with the rapid and complete correction of the anaemia. 
The overall incidence of lymph node enlargement in the 250 treated patients is $3.6 \%$ but it rises to $7.6 \%$ in the women.

Painful enlargement of draining lymph nodes has been reported after multiple intramuscular injections of iron-dextran. Ben-Ishay (1961) described 11 such cases with generalized symptoms as well. Ten of the 11 patients were also women but none had a biopsy of the enlarged nodes.

The pathogenesis of the enlargement is unknown. The iron deposited in the nodes was probably the injected material and not haemosiderin. Iron as such can be incriminated for the localized enlargement of the draining nodes after intramuscular injections due to extreme iron concentration. In our cases the serum iron concentration at the time of the node enlargement had fallen well below $1,000 \mu \mathrm{g} \%$.

The histology is not specific for either a hypersensitivity reaction or an antibody response to injected antigenic material. However, since the histological changes, especially after injection of an antigen and production of antibody by the lymph node, do not last long, it may be conceivable that when the node was removed some of the changes had already regressed.

In view of the findings of Turk (1967) in experimental animals, this lymph node, with the exception of the absence of germinal centres in the cortex, shows somewhat similar changes to those seen in animals after injection of pneumonococcal polysaccharide. Alternatively the changes seen may be completely non-specific due to the presence of the iron complex itself in large amounts.

Kabat and Berg (1953) and Maurer (1953) have shown that dextran is antigenic in man. They have also found that some people had naturally occurring anti-dextran antibodies without having previously received dextran.

These findings support the hypothesis that the reported lymph node enlargement and histological 0 changes and the clinical syndrome are closely related to an antigen-antibody reaction. Most probably there is no antibody present before the administration of the iron-dextran complex but it is formed afterwards, and this explains the appearance of the general symptoms several days after its infusion. Perhaps in patient A.M. the appearance of the general symptoms almost immediately after the infusion may indicate the preexistence of naturally occurring anti-dextran antibodies.

The reason for the appearance of this complication in women only is not clear.

We wish to thank Dr D. Georgiadou for doing the histology of the lymph node.

\section{REFERENCES}

Basu, S. K. (1963). Lancet, 1, 1430.

Ben-Ishay, D. (1961). Ibid., 1, 476.

Bonnar, J. (1966). In XI Congress of the International Society of Haematology, Sydney. Volume of Abstracts of papers, page 53.

Kabat, E. A., and Berg, D. (1953). J. Immunol., 70, 514.

Marchasin, S., and Wallerstein, R. O. (1964). Blood, 23, 354.

Maschas, H., Constantoulakis, M., Chilaiditis, G., Floras, A., and Makkous, A. (1966). Sém. Hôp. Paris, 42, 1067.

Maurer, P. H. (1953). Proc. Soc. exp. Biol. (N. Y.), 83, 879.

Symposium, (1967). Treatment of Iron Deficiency Anaemia in Obstetrics and Gynaecology with Total Dose Infusion of Iron- $\overrightarrow{\bar{O}}$ Dextran. London. Chairman of the Symposium Sir Hector $\overline{3}$ MacLennan, held in London 22nd March 1967. Symposium Sponsored by Fisons Pharmaceuticals Ltd., Loughborough, Leicestershire.

Theodoropoulos, G., Constantoulakis, M., Zevgolatis, C., and Phocas E. (1966). Nosokom. Chron., 28, 295.

Turk, J. L. (1967). Brit. med. Bull., 23, 3. 